\title{
La neolitización del nordeste de la Península Ibérica: datos radiocarbónicos y culturales de los asentamientos al aire libre del Penedès
}

\author{
The neolithisation process in the northeastern Iberian Peninsula: radiocarbon and \\ cultural data from open-air sites in Penedès
}

F. Xavier Oms (*)
Xavier Esteve (*)
Josep Mestres (**)
Patricia Martín $(* * *)$
Haidé Martins $(* * * *)$

\section{RESUMEN}

En este trabajo se presentan datos inéditos acerca de tres asentamientos al aire libre situados en el llano prelitoral del Penedès (Barcelona) con ocupaciones del Neolítico inicial: el hábitat de Les Guixeres de Vilobí y las agrupaciones de silos-fosas de La Serreta y el Mas d'en Boixos. Se estudian sus materiales cerámicos y 5 fechas radiocarbónicas inéditas en el marco de la discusión sobre el Neolítico antiguo del nordeste de la Península Ibérica y de la neolitización del Mediterráneo occidental. Se propone que esta zona fue clave y con cierta densidad poblacional durante las primeras fases neolíticas y comparable a enclaves emblemáticos como el cercano valle del Llobregat (Garraf-llano de Barcelona y Vallès) o la zona alicantina. La antigüedad de las fechas obtenidas aporta nuevos datos al debate acerca del primer neolítico en la Península Ibérica.

\footnotetext{
ABSTRACT

The work now presented puts forward new data on three open-air sites located in Penedès (Barcelona, Cat-

(*) Seminari d'Estudis i Recerques Prehistòriques (SERP), Universitat de Barcelona. C/ Montalegre 6-8. 08001 Barcelona, España. Correos e.: xavieroms@gmail.com; xesteveg@gmail.com

(**) VINSEUM, Museu de les Cultures del Vi de Vilafranca del Penedès-Catalunya. Plaza Jaume I. 08720 Vilafranca del Penedès, España. Correo e.: josepmestres@hotmail.com

$(* * *)$ Institut Català d'Autoecologia Humana i Evolució Social (IPHES). C/ Marcel·lí Domingo s/n. 43007 Tarragona, España. Correo.e: patrimr9@gmail.com

(****) Department of Archaeology and Anthropology, University of Bristol. 43 Woodland Road. Bristol BS8 1UU, United Kingdom. Correo.e: arhmdcm@bristol.ac.uk

Recibido 11-XII-2012; aceptado 18-II-2013.
}

alan Coastal Depression) with Early Neolithic occupation phases: the settlement of Les Guixeres de Vilobi and the pit complexes of La Serreta and Mas d'en Boixos. The sites' pottery assemblages were analyzed and, for the first time, 5 short-lived samples were radiocarbon dated to collaborate in the discussion of the current knowledge on the Early Neolithic of NE Iberia and the broader Western Mediterranean. It suggests this area played an important role during the earliest phases of the Neolithic together with other key areas such as the Llobregat valley or the Alicante region. The chronology obtained enabled us to discuss the earliest Neolithic of the Iberian Peninsula.

Palabras clave: Cerámica cardial; Neolítico antiguo; C14; Penedès; Mediterráneo occidental.

Key words: Cardial pottery; Early Neolithic; C14; Penedès; Western mediterranean.

\section{INTRODUCCIÓN AL MEDIO GEOGRÁFICO}

El Penedès es uno de los enclaves con mayor concentración de yacimientos del Neolítico antiguo Cardial de la Península Ibérica. Desde la publicación en 1932 de las excavaciones de Martí Grivé en l'Esquerda de les Roques del Pany, se han documentado un total de 26 al aire libre o en cueva/abrigo. La situación geográfica y el contexto geomorfológico de la zona son los principales argumentos para esta densa ocupación. No se dispone de datos estratigráficos o publicados de manera extensa de la mayoría de ellos, pero aun 
así han trascendido como puntos clave para la comprensión del proceso de neolitización del nordeste peninsular, vinculándose habitualmente con el llamado grupo del Llobregat (Bernabéu 1999; Martín et al. 2010).

El estudio de los materiales cerámicos ha contado con el rico registro del hábitat de Les Guixeres de Vilobí, en parte publicado a principios de los 1980 (Mestres 1981-1982). En el resto de los yacimientos (silos/fosas), con menor número de efectivos, los fragmentos de diferentes estructuras se han reunido para contar con un conjunto más representativo estadísticamente. Las 5 fechas radiocarbónicas publicadas en este trabajo son las primeras que se obtienen para este territorio del Neolítico antiguo. Sobre esta base se pretende actualizar y validar los datos existentes en la zona integrándolos en las discusiones sobre la neolitización peninsular.

El Penedès forma parte de la llamada Depresión Prelitoral Catalana, una región natural e histórica, extendida por el litoral y prelitoral central catalán, hasta el Camp de Tarragona. La forman las actuales comarcas del Alt y Baix Penedès y del Garraf. Está integrada en el Sistema Mediterráneo Catalán, cuyas cuatro unidades de relieve son una cordillera prelitoral, una depresión prelitoral, una cordillera litoral o de marina y un llano litoral discontinuo. El artículo se centra en la depresión cuyas cuencas drenan el río Llobregat, a través de su afluente el Anoia, el río Foix y otros menores. Tiene unos $42 \mathrm{~km}$ de longitud en sentido NE-SO y unos $15 \mathrm{~km}$ de anchura máxima. Es una llanura de relieve bastante irregular donde se alternan superficies horizontales con pequeñas elevaciones, a menudo recortadas por una ramificada red fluvial. Con la división provincial el Penedès, y la depresión citada, quedó repartido entre las de Barcelona (Alt Penedès y Garraf) y Tarragona (Baix Penedès) (Fig. 1).

Los yacimientos considerados en el presente artículo se localizan en el territorio siguiendo patrones frecuentes durante la prehistoria reciente. Al aire libre se centran en la Depresión Prelitoral entre 180-340 m.s.n.m. en torno al núcleo de Vilafranca del Penedès (La Serreta y Vinya d'en Pau) sobre todo en la vertiente de suaves elevaciones (303 m.s.n.m.) de la montaña de Sant Pau (Mas d'en Boixos). Las cuevas y abrigos se hallan mucho más dispersos por el territorio pero, en mayor medida, en la Cordillera Prelitoral que

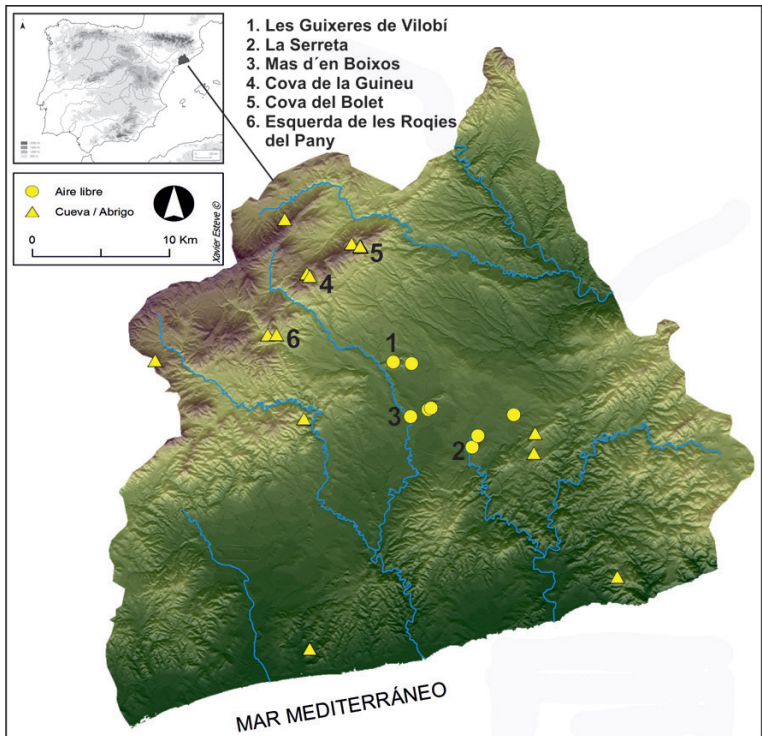

Fig. 1. Localización de la comarca del Penedès en la Península Ibérica y de los asentamientos con cerámicas cardiales del Neolítico antiguo (numerados los citados en el texto), sobre imagen MDT30 (ICC).

en la Litoral a alturas de hasta 800 m.s.n.m. Los asentamientos son heterogéneos, ya que las condiciones de habitabilidad de las cavidades, a excepción de las Covas del Bolet y la Guineu, son escasas

\section{BREVE HISTORIA DE LA INVESTIGACIÓN EN EL PENEDÈS}

Las primeras noticias sobre la fase neolítica se relacionan con la publicación de la excavación de Martí Grivé (Baldellou et al. 1989) del emblemático y rico yacimiento de l'Esquerda de les Roques del Pany (Torrelles de Foix) en 1932. Esos trabajos permiten una primera aproximación cronológica al fenómeno cardial, al situar sus elementos materiales en un estrato subyacente a un paquete con cerámicas carenadas (argáricas según Grivé) y campaniformes.

En 1972 Vicente Baldellou llevó a cabo una campaña arqueológica en la Cova del Bolet (Mediona). Realizó 5 sondeos de $4 \mathrm{~m}^{2}$, cuatro en el vestíbulo y otro en el exterior. Solo se recuperó un efectivo cerámico in situ de los niveles más antiguos con una decoración inciso-impresa (E3 estrato II) que recuerda a un horizonte Epicardial. Los restantes efectivos parecen corresponder a 
ocupaciones postcardiales (Baldellou 19791980). Sin embargo se conoce un lote de materiales cardiales muy importante de este yacimiento (Giró 1947-1948), procedente de excavaciones previas no reguladas.

Les Guixeres de Vilobí (Sant Martí Sarroca) es un asentamiento al aire libre emblemático de esta zona, situado en una pequeña elevación de materiales cretácicos a 330 m.s.n.m. Fue excavado durante 1974 y de 1981 a 1984 con metodología moderna: cuadrícula, representación tridimensional del material arqueológico, documentación de estructuras, etc. Se identificaron numerosos estratos y estructuras que se resumen en tres fases de ocupación que comprenden todo el Neolítico antiguo y el Neolítico medio inicial (Cardial, Epicardial y Postcardial Molinot). Probablemente se trate del primer hábitat estricto (campos de silos aparte) de cronología cardial (Baldellou y Mestres 1981; Mestres 1987) definido en la Península Ibérica. Más adelante se profundizará en su análisis.

La Cova de la Guineu (Font-rubí) está situada a 800 m.s.n.m. en la cima de la Plana Pineda, al occidente del llano del Penedès. En proceso de excavación desde 1983, su larga secuencia ocupacional se inicia en el Epipaleolítico microlaminar (Equip Guineu 1995; Bergadà et al. 2005). El nivel del Neolítico antiguo Postcardial de facies Molinot parece romper uno previo con cerámicas cardiales lo que, unido a la intensa actuación de animales cavadores, ha impedido hasta el momento documentar in situ la ocupación del Neo- lítico antiguo Cardial. El conjunto de cerámicas impresas es muy importante y se asocia a una interesante industria ósea con punzones, cucharas y anillos. A. Cebrià, J. I. Morales y X. Oms prevén excavar en los próximos años los niveles cardiales intactos aparecidos bajo un gran bloque desprendido de la visera (Fig. 2).

La arqueología preventiva desde la década de los 1990 ha permitido documentar nuevos asentamientos con vestigios cardiales o epicardiales. Presentan un máximo de 9 estructuras negativas de tipo silo/fosa, pequeñas y más o menos cercanas. Algunos cuentan con memoria de excavación y otros han sido parcialmente publicados. Destacamos el Mas d'en Boixos (Pacs del Penedès) donde entre 1999 y 2008 se han excavado en campañas preventivas un total de 459 con grandes concentraciones de estructuras postcardiales, de la Edad del Bronce inicial y de la Primera Edad del Hierro. Las identificadas como E299 y E337 tienen cerámicas cardiales. En estos últimos años se han excavado, además, tres silos cardiales y uno epicardial en La Serreta (Vilafranca del Penedès) (Esteve et al. 2012) y otro cardial en la Vinya d'en Pau en el transcurso de obras viarias a su paso por Vilafranca del Penedès.

Para este trabajo nos hemos centrado en los asentamientos de Les Guixeres de Vilobí, La Serreta y Mas d'en Boixos por tratarse de yacimientos al aire libre con datos mayormente inéditos. Enclaves emblemáticos del Penedès como la Cova de la Guineu, la Cova del Bolet y l'Esquerda de les Roques del Pany serán tratados en futuros
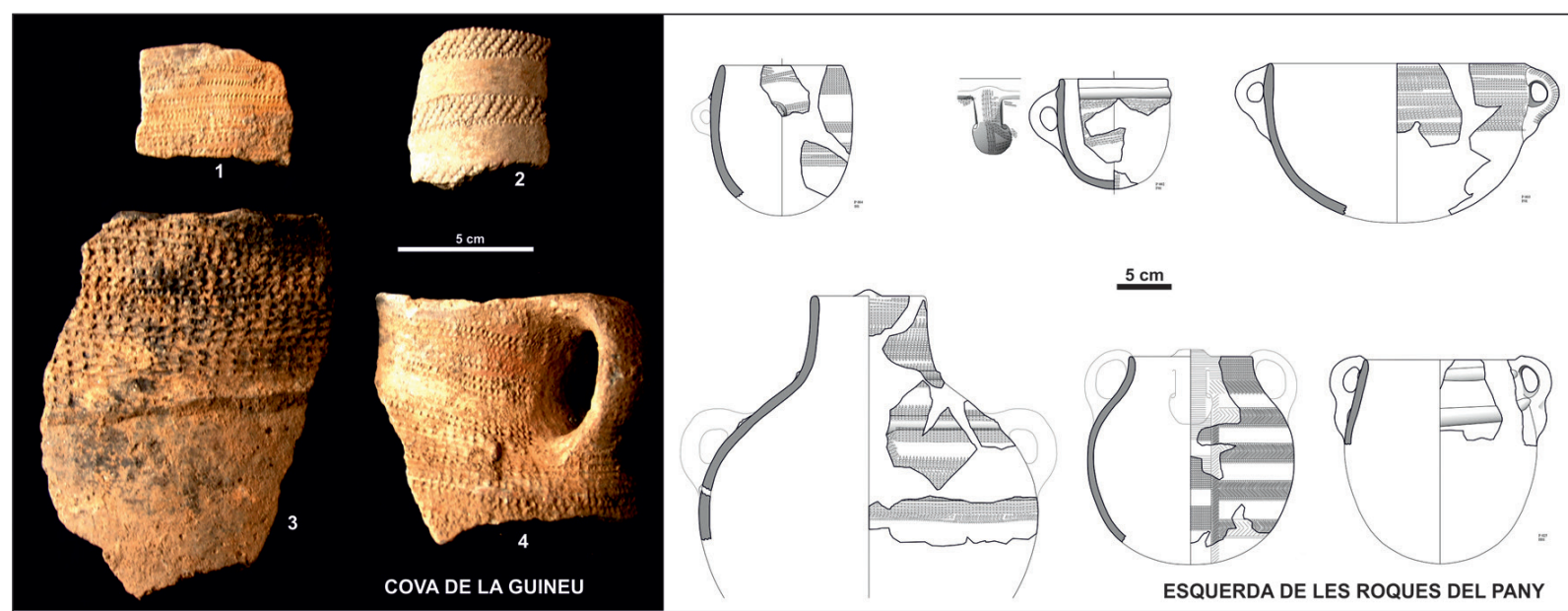

Fig. 2. Cerámicas cardiales del Neolítico Cardial del Penedès: Cova de la Guineu y de l'Esquerda de les Roques del Pany (foto X. Oms; dibujos J. Mestres).

Trab. prehist., 71, N. ${ }^{\circ}$ 1, enero-junio 2014, pp. 42-55, ISSN: 0082-5638 doi: $10.3989 /$ tp.2014.12123 


\begin{tabular}{|c|c|c|c|c|c|c|c|}
\hline Yaci & $\mathbf{U E}$ & Mu & a & BP & atio & Cal BC $1 \sigma$ & Cal BC 2 $\sigma$ \\
\hline $\mathrm{La}$ & & AIII & & 0 & & & \\
\hline La Serı & 56) & Arbut & 62 & 649 & -24.0 & 550 & 5550 \\
\hline La Serreta & E79 (7901) & Arbutus & Beta-280866 & 6420 & -23.3 & 5460 & 551 \\
\hline Les Guir & Hori & Ovis & $\mathrm{Ox}$ & 665 & -19.3 & 562 & $5660-5500$ \\
\hline Les Gui & onte $\mathrm{A}$ & $O v i$ & -26069 & $6458 \pm 38$ & -19.1 & $5470-5390$ & $5510-5350$ \\
\hline
\end{tabular}

Tab. 1. Dataciones radiocarbónicas de los asentamientos al aire libre del Penedès (nordeste de la Península Ibérica) con cerámica cardial, estudiados en el artículo (calibraciones mediante el software CalPal'07 curva Hulú Weninger et al. 2011).

trabajos de contextualización de los ámbitos de la sierra prelitoral.

\section{CRONOLOGÍA RADIOCARBÓNICA: NUEVOS DATOS}

Para este estudio se han fechado por $A M S$ muestras de cada una de las estructuras cardiales de La Serreta. El material seleccionado fue carbón recogido manualmente durante los trabajos arqueológicos por ausencia de restos zooarqueológicos o carpológicos por flotación. Se intentó la identificación taxonómica de las muestras para elegir las consideradas de vida corta. Solo se logró en las estructuras E61 y E79, donde se utilizaron sendos carbones de ramitas de Arbutus unedo, un taxón que consideramos más adecuado para la datación que otros potencialmente más longevos (Quercus p.ej.). De la estructura E59 se escogió un fragmento de Angiosperma indeterminada.

Del Horizonte A de Les Guixeres de Vilobí proceden otras dos fechas, esta vez, sobre restos óseos de Ovis aries: OxA-26068 (cuadro V-G49 n. ${ }^{\circ}$ 39) se obtuvo a partir de un molar y OxA26069 (V-K32 n. ${ }^{\circ}$ 87) de un metápodo.

Los silos de Mas d'en Boixos no se pudieron datar porque durante su excavación no se recogieron macrorestos vegetales ni se flotó sedimento (1). Se prefirió no datar restos malacológicos (Tab. 1).

(1) Vidal, A. 2007: Memòria cientifica de la intervenció arqueolòica al jaciment de Mas d'en Boixos (Pacs del Penedès, Alt Penedès). Octubre de 2004 - Febrer de 2005. Arxiu Servei d'Arqueologia i Paleontologia, Generalitat de Catalunya, Barcelona. Consulta mayo 2011.
Las fechas de La Serreta y OxA-26069 de Les Guixeres muestran cierta homogeneidad. En cambio, OxA-26068 de Les Guixeres es más antigua y sólo coincide en los extremos de la horquilla (a $2 \sigma$ ) con las anteriores. El contexto arqueológico de procedencia de la muestra (estratos basales de la estructura principal) y su naturaleza (vida corta y doméstica) dotan de alta fiabilidad a la datación.

Las fechas obtenidas para ambos yacimientos concuerdan de manera óptima con las horquillas asumibles para el Neolítico inicial catalán (Morales et al. 2010) y suponen las primeras obtenidas para esta cronología en el Penedès.

\section{YACIMIENTOS ESTUDIADOS: ESTRATIGRAFÍA, ESTRUCTURAS Y CONTENIDO ARQUEOLÓGICO}

Como recordaremos, en la zona del Penedès se conocen numerosos yacimientos pertenecientes a las primeras fases neolíticas, de los cuales un número importante son al aire libre. Caracterizamos los incluidos en este estudio: Les Guixeres de Vilobí, un hábitat complejo con gran estructura central, fosas y agujeros de poste y los de La Serreta y Mas d'en Boixos con agrupaciones de estructuras negativas de tipo silo.

La superficie excavada en Les Guixeres de Vilobí (Sant Martí Sarroca) fue de $127 \mathrm{~m}^{2}$. Destacamos una gran estructura negativa de $12 \mathrm{~m}$ de longitud máxima y $6 \mathrm{~m}$ de ancho, con una potencia máxima de $60 \mathrm{~cm}$ en la zona central. La funcionalidad primaria de esta depresión no se ha definido. Estaba colmatada por restos arqueológicos heterogéneos, a modo de hábitat abandonado o simple basurero. En su relleno se documentó una estructura de combustión de $150 \mathrm{~cm}$ de diá- 
metro máximo y $25 \mathrm{~cm}$ de potencia. En algunos tramos del contacto con el substrato de yesos, estaban incrustados numerosos fragmentos cerámicos planos. Esta gran estructura se relacionaba con otras tres ovales, alineadas, de 50-60 cm de diámetro y $40 \mathrm{~cm}$ de potencia, rellenas de bloques e interpretadas como agujeros de poste de gran tamaño, así como con una cuarta, próxima, de tipo silo.

La secuencia estratigráfica de todo el yacimiento tiene escasa potencia $(70 \mathrm{~cm}$ en la zona sin estructuras negativas) y es bastante homogé- nea y relativamente simple. Se reduce a tres fases: un primer horizonte (C) con materiales pertenecientes al Neolítico antiguo Postcardial con cerámicas peinadas (tipo Molinot); un segundo horizonte (B), poco potente, con materiales impresos, incisos y plásticos que se pueden situar bien en un Neolítico Epicardial y un horizonte basal (A) de unos $35 \mathrm{~cm}$ de potencia con un rico conjunto de materiales cardiales (Baldellou y Mestres 1981; Mestres 1981-82, 1987) (Fig. 3).

Según el estudio arqueozoológico de las fases más antiguas, predominan los ovicápridos $(56,6 \%)$

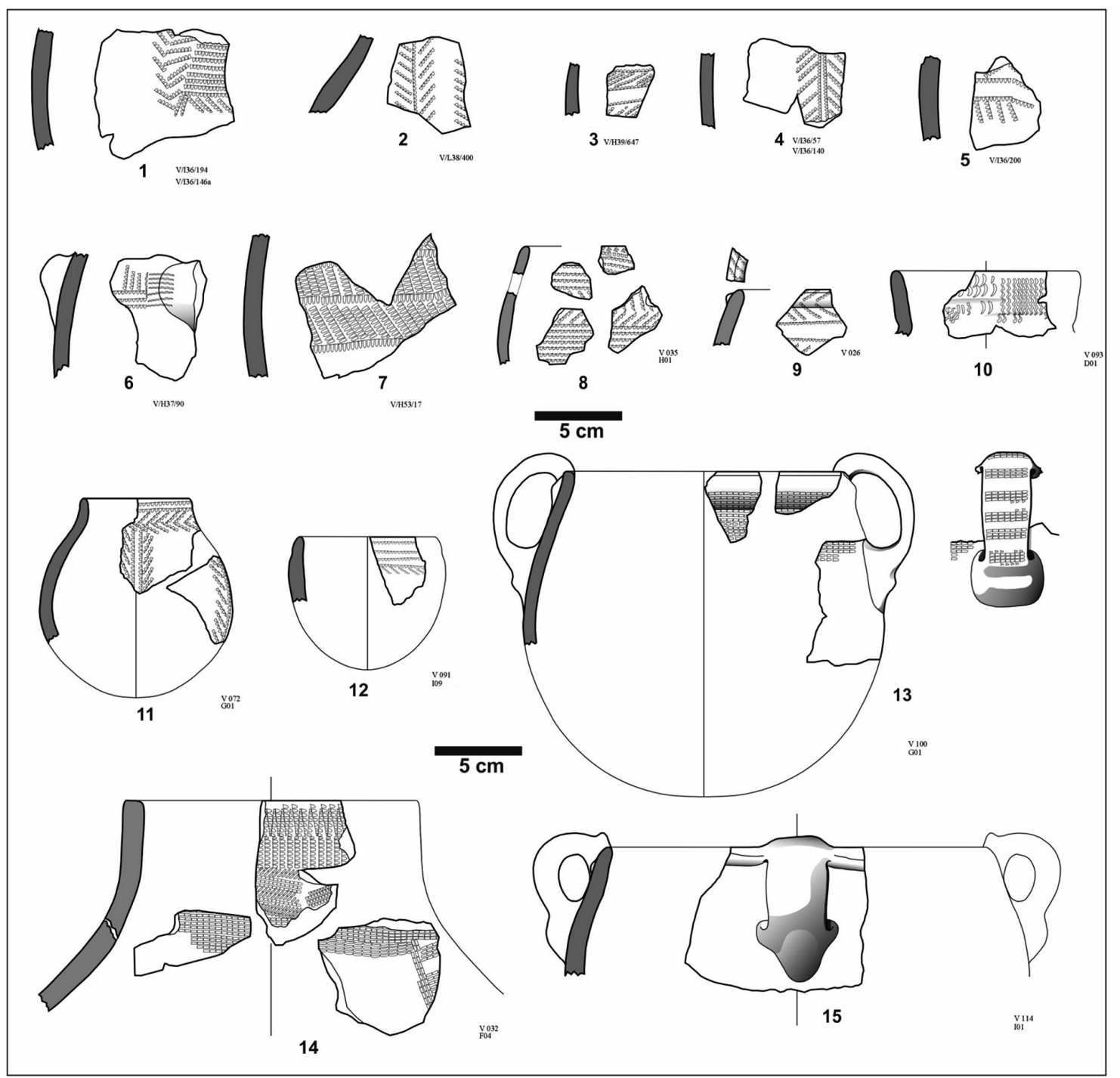

Fig. 3. Cerámicas cardiales del Neolítico Cardial de la comarca del Penedès de Les Guixeres de Vilobí, Horizonte A (dibujo J. Mestres). 
frente a suidos y bóvidos. Los taxones salvajes, muy minoritarios, se reducen al ciervo $(3,77 \%)$ y quizá el conejo (c. 20\%) (Miró 1992; Nadal et al. 1999). La industria lítica del Horizonte A es muy numerosa (2073 restos). En su mayoría está realizada sobre sílex, local y foráneo, con presencia puntual de cuarzo, jaspe y cristal de roca. Destacamos la neta superioridad de las lascas $(75,1 \%)$ sobre las láminas $(24,8 \%)$. Predominan como soportes las lascas cortas estrechas y cortas anchas. Los núcleos poliédricos y prismáticos (c. 65\%) son los más representados. Las piezas retocadas son 224: grupo de muescas/denticulados $(27,23 \%)$, láminas $(23,66 \%)$, perforadores $(12,5 \%)$ y geométricos $(10,27 \%)$. Los 23 geométricos son 1 segmento con retoque abrupto, 4 triángulos con retoque plano bifacial y 18 trapecios simétricos, asimétricos o con lados cóncavos, con retoques planos o abruptos (Mestres 1987).

La Serreta (Vilafranca del Penedès) es otro asentamiento al aire libre con 89 estructuras negativas, la mayoría de tipo fosa/silo y pertenecientes al Neolítico Postcardial Molinot y al Neolítico medio (Esteve et al. 2012) (Fig. 4). Las E59, E61 y E79, muy cercanas, de donde proceden las muestras fechadas, son todas estructuras de tipo silo. Contienen cerámicas cardiales y se hallan a cotas entre 180-190 m.s.n.m.

La E59 tiene una abertura documentada de $110 \mathrm{~cm}$ y una profundidad de $15 \mathrm{~cm}$. El perfil, con una erosión importante, es de tendencia abierta. Se identificaron dos niveles de relleno. Uno estaba repleto de clastos centimétricos quemados. El otro parecía poco antropizado y probablemente era una pared caida. La estructura contenía escasa industria lítica, un fragmento de núcleo y láminas retocadas en sílex, así como fragmentos de macroutillaje. La E61 tenía un diámetro máximo de $85 \mathrm{~cm}$, una potencia entre $35-45 \mathrm{~cm}$ y sección troncocónica, afectada en un tercio por un servicio telefónico reciente. Se diferenciaron hasta seis unidades estratigráficas, en su gran mayoría muy orgánicas. A pesar de ello, no se recuperaron restos faunísticos y apenas líticos (algún núcleo y láminas en sílex y jaspe). La E79 tenía un diámetro máximo de $135 \mathrm{~cm}$ y una profundidad conservada de unos $30 \mathrm{~cm}$. Una de las tres unidades estratigráficas definida era rica en bloques y clastos angulosos, algunos quemados. Entre los materiales no cerámicos destacan fragmentos de molino y alguna lámina en cristal de roca.
Del asentamiento de Mas d'en Boixos (Pacs del Penedès) se conocen hasta cinco estructuras con materiales cardiales (Fig. 4). Solo en dos (campaña de Álex Vidal en 2004) no están mezclados con otros de filiación Epicardial y Postcardial. Se trata de la E-299, un silo globular con una potencia de $66 \mathrm{~cm}$ y un diámetro máximo de $100 \mathrm{~cm}$ y de la E-337, una cubeta de $22 \mathrm{~cm}$ de potencia y un diámetro máximo de $93 \mathrm{~cm}$. Además de los restos cerámicos, en la E-299 se recuperaron tres conchas de Cardium y algunas lascas de sílex y en la E-337 un Cardium perforado y un núcleo poliédrico de jaspe. No se hallaron restos arqueozoológicos, ni se conservó sedimento para su flotación (2).

\section{LA DECORACIÓN CERÁMICA EN LOS YACIMIENTOS ESTUDIADOS}

El registro cerámico disponible está muy fracturado. Nos hemos aproximado al número mínimo de vasos a partir de criterios macroscópicos (morfología, decoración, cocción, acabados, etc.). En este contexto el recuento de fragmentos decorados depende de que nos refiramos a su número total o al de vasos identificados, ya que varios con la misma o distinta decoración pueden pertenecer al mismo vaso. Lo segundo explica que el total de decoraciones suela ser más elevado que el de fragmentos. En este trabajo, y para poder comparar nuestros resultados con los publicados por la gran mayoría de los colegas en Cataluña, definiremos las decoraciones por número de fragmentos.

Les Guixeres de Vilobí totaliza 1524 fragmentos en el Horizonte A, de los cuales 332 $(21,8 \%)$ están decorados. La impresión cardial supera algo el $81 \%$ del conjunto. Aparece sobre un cordón en el 3,91\% de los casos. Un fragmento está decorado con gradina $(0,4 \%)$. Los cordones lisos de escaso relieve (en posición horizontal o vertical) completan con un $14,45 \%$ los efectivos. La elevada rotura dificulta el cálculo exacto del número mínimo de vasos (supuesto en más de 1200, casi 300 decorados), así como de los decorados con más de una técnica o motivo. Las estructuras negativas ligadas al Horizonte A disponen de 43 efectivos, de los cuales sólo 2 se

(2) Véase n. 1. 


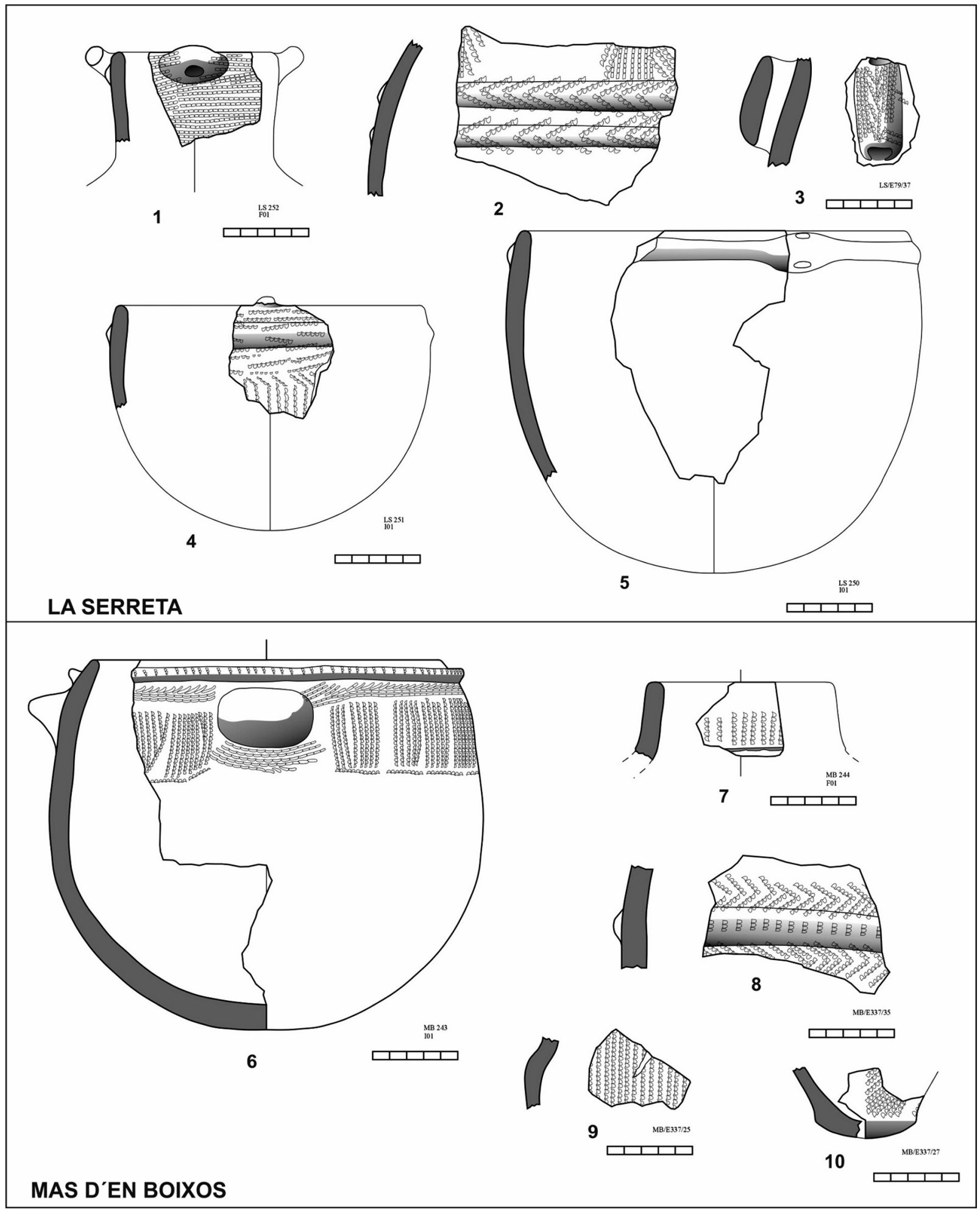

Fig. 4. Cerámicas cardiales del Neolítico Cardial de la comarca del Penedès: La Serreta y de Mas d'en Boixos (dibujo J. Mestres). 


\begin{tabular}{|l|c|c|c|c|c|c|}
\hline & A & B & C & D & E & TOTAL \\
\hline Les Guixeres A & $\begin{array}{c}269(81,02) \\
216^{*}\end{array}$ & $\begin{array}{c}13(3,91) \\
11^{*}\end{array}$ & $1(0,4)$ & $\begin{array}{c}48(14,45) \\
36^{*}\end{array}$ & $1(0,4)$ & $\begin{array}{c}332 \\
265^{*}+7^{*}\end{array}$ \\
\hline La Serreta E59 & 2 & - & - & 4 & - & 6 \\
\hline La Serreta E61 & 4 & 6 & - & 2 & 1 & 13 \\
\hline La Serreta E79 & 3 & - & - & 1 & - & 4 \\
\hline La Serreta TOTAL & $\begin{array}{c}(39,1) \\
6^{*}\end{array}$ & $\begin{array}{c}6(26,08) \\
2^{*}\end{array}$ & 0 & $\begin{array}{c}7(30,43) \\
5^{*}\end{array}$ & $1(4,34)$ & $\begin{array}{c}23 \\
14^{*}\end{array}$ \\
\hline Mas d'en Boixos E299 & 7 & & - & - & - & 7 \\
\hline Mas d'en Boixos E337 & 7 & 4 & - & 1 & - & 12 \\
\hline TOTAL Mas d'en Boixos & $\begin{array}{c}14(73,68) \\
6^{*}\end{array}$ & $\begin{array}{c}4(21,05) \\
2^{*}\end{array}$ & 0 & $1(5,26)$ & 0 & $\begin{array}{c}19 \\
{ }^{*}\end{array}$ \\
\hline TOTAL & 292 & 23 & 1 & 56 & 2 & 377 \\
\hline
\end{tabular}

Tab. 2. Totales y porcentajes de tipos de decoración cerámica de los 3 yacimientos analizados del Neolítico antiguo del Penedès y número mínimo* de recipientes calculados: A cardial; $\mathrm{B}$ cardial sobre cordón; C impresa; D cordón liso; E cordón impreso. Son exclusivos de Les Guixeres A las nervaduras (7 fragmentos atribuidos a 6 vasos) y la gradina (1 ejemplar).

ornamentan (cordones lisos). Por su escasa cuantía excluimos este conjunto de los recuentos (Tab. 2).

En las tres estructuras tipo silo de La Serreta, se han recuperado 72 fragmentos cerámicos (39,1\% decorados), correspondientes a un mínimo de 51 vasos (14 decorados). Los efectivos con decoración cardial (sobre pared y cordón) suponen casi el $65 \%$ del total. Los cordones lisos superan el 30\%. Otras decoraciones tienen poca representatividad.

Del silo E-299 y de la cubeta E-337 de Mas d'en Boixos proceden 57 fragmentos cerámicos, asignados a un mínimo de 21 vasos, de los cuales 19 (33,3\%) están decorados (correspondientes a 9 vasos). El cardial aparece en 18 de ellos sobre pared $(73,68 \%)$ o cordón $(21,05 \%)$. Solo uno tiene cordones lisos aplicados.

Las características de los motivos y las técnicas del conjunto, salvo las excepciones que comentaremos, son las más clásicas del escenario Cardial zonado o franco-ibérico. Predominan las franjas horizontales de impresiones cardiales, a veces intermitentes y de diferente grosor, desde finas y esbeltas a gruesas formaciones que cubren todo el tercio superior del vaso (Figs. 2: 3 - 4 y 4: 1). Son franjas simples, únicas o dobles paralelas, y en contadas ocasiones más numerosas (Esquerda de les Roques del Pany o vaso 2 de Guineu) (Fig. 2). A veces las forman espigas horizontales o de ellas se desprenden impresiones sueltas en flecos oblicuos. Por último, a partir de las franjas horizontales cardiales se desarrollan otras verticales, con motivos simples o complejos en espiga. Las franjas cortadas formando metopas no son habituales, pero existen en casi todos los conjuntos cardiales del Penedès (en este estudio Les Guixeres Horizonte A, Fig. 3: 1; Mas d'en Boixos, Fig. 4: 6).

En general, la decoración cardial se dispone en posición o perpendicular con el borde dentado u oblicua. La impresión pivotante de corto recorrido es ocasional (Les Guixeres, Fig. 3: 14). El cardial arrastrado es muy minoritario en estos conjuntos. Aparece en Mas d'en Boixos E-299, en un efectivo de La Serreta E-59 y en otros contados de Les Guixeres A. Cuando las impresiones cardiales se dan sobre cordón horizontal, suelen oponerse oblicuamente como espigas parciales (Figs. 4: 2 y 8). Los cordones lisos (casi siempre aplicados) son de escaso relieve tienen posición horizontal (mayoritaria), vertical o arciforme.

Las impresiones no cardiales son escasas. Se hicieron con objetos de punta roma o media caña. Las incisiones y los acanalados están totalmente ausentes. Solo en el fragmento 10 de Les Guixeres A (Fig. 3) la técnica y disposición de las impresiones recuerda horizontes ligures: impresiones verticales desordenadas del frente 
dentado del Cardium. Unos pocos fragmentos conservan ocre en superficie (Les Guixeres A, Esquerda de les Roques del Pany, Mas d'en Boixos E-299) sin que puedan considerarse pintados (Mestres 1992).

\section{CONTEXTUALIZACIÓN CRONOLÓGICO-ESTILÍSTICA}

El grupo de yacimientos de la fase Cardial situados en las zonas litoral y prelitoral del nordeste de la Península Ibérica presenta una relativa homogeneidad, ya conocida en el grupo Cardial franco-ibérico (Manen 2002). La problemática más acusada es su periodización interna, ya que durante el lapso c. 6600-6100 BP se detecta cierta continuidad de las técnicas cardiales en muchos yacimientos del valle del Llobregat, mientras que en otros territorios, a partir de $6250 \mathrm{BP}$, otros tipos decorativos hacen acto de presencia (Oms et al. 2012).

En el llano del Penedès solo se conocen datos de la fase más antigua del período cardial. Llamada "Cardial antiguo" (Martin et al. 2010) o "Fase I" (Manen 2002), se caracteriza por altos porcentajes de decoración cardial (c. 60\%) de diferente tipo (crochet, arrastrado, etc.), seguidos por los de las aplicaciones plásticas (casi en el $90 \%$ también decorados con impresiones) y por un uso esporádico de impresiones de instrumento. Esta representación masiva de lo cardial se cumple en Les Guixeres de Vilobí (c. 85\%), La Serreta (c. 65\%) y Mas d'en Boixos (c. 90\%). A su vez, esta dinámica es habitual en ámbitos cardiales cercanos (Fig. 5) como la Cova de Can Sadurní en el Garraf (Edo et al. 2011), Sant Pau del Camp en el llano de Barcelona (Molist et al. 2009) o la Cova del Frare en el Vallès (Manen 2002). En cambio, en la zona provenzal y en Alicante, los porcentajes son menores en beneficio de otras matrices (Bernabéu 1989; Bernabéu y Molina 2009).

Las morfologías de los vasos, los temas decorativos y las técnicas predominantes, con ciertas variaciones geográficas, se cumplen de manera relativamente homogénea para el periodo Cardial (Bernabéu et al. 2011) en las producciones del Penedès. El escenario de los motivos y técnicas varía a medida que avanza el periodo. Las producciones con muy buenos acabados y composiciones cardiales simples (aunque puntualmente barrocas) con fechas radiocarbónicas antiguas (Les Guixeres A, Cova de Can Sadurní c18, El Cavet) se confrontan con las de los yacimientos más recientes que poseen una gama más variada

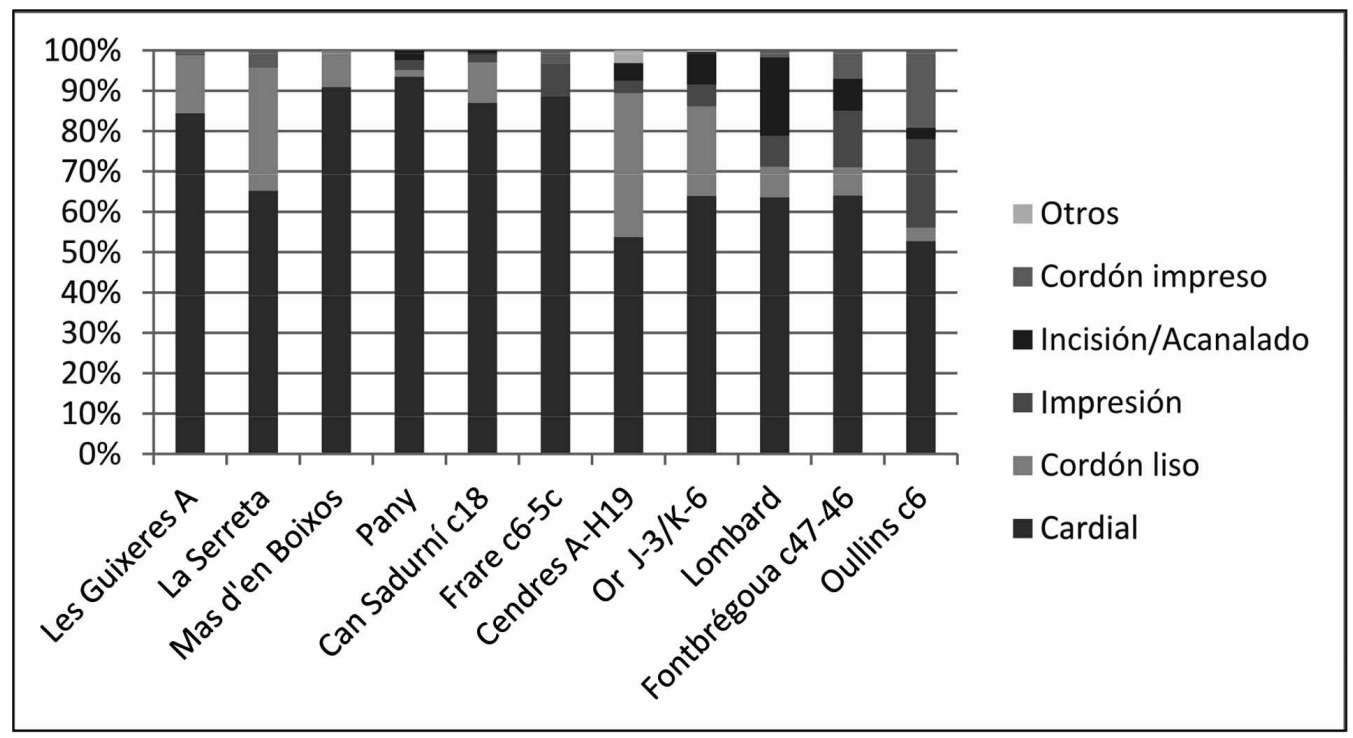

Fig. 5. Tipos decorativos porcentuales por número de fragmentos decorados de contextos mediterráneos españoles y franceses con cerámicas cardiales (según Bernabéu 1989; Manen 2002; Bernabéu y Molina 2009; Edo et al. 2011). 
de técnicas impresas cardiales y combinaciones complejas. Pensamos en la Draga (Bosch et al. 2011), en Sant Pau del Camp (Molist et al. 2009) o en la Cova de la Font Major (excavación de Marta Fontanals y Patrica Martín en 2011), donde se dan las técnicas de arrastre, la impresión con natis y la profusión de la gradina, técnicas poco frecuentes en las fases más antiguas.

\section{LOS HORIZONTES ANTIGUOS DEL NEOLÍTICO INICIAL: DISCUSIÓN Y RECAPITULACIÓN}

Las fechas radiocarbónicas existentes sobre las primeras fases neolíticas y sus correspondientes grupos culturales dificultan la incorporación del registro material del Horizonte A de Les Guixeres con una fecha $6655 \pm 45$ BP (OxA-26068). Según las dataciones actuales los conjuntos cardiales netos no deberían superar una antigüedad c. $6500 \mathrm{BP}$ sobre muestras de vida corta al oeste de la zona provenzal (Binder et al. 1993; Binder y Maggi 2001; Binder y Sénépart 2010; Molina et al. 2011). La fecha del Horizonte A de Les Guixeres podría indicar la presencia de una fase precardial amortizada y mezclada con los materiales cardiales o la posible coexistencia de estos últimos con elementos precardiales: los escasos fragmentos cerámicos antes citados. Sin embargo la total sintonía de los materiales de Les Guixeres A con los estándares clásicos cardiales y el carácter anecdótico y poco significativo de los discordantes impiden sustentar tales opciones con suficiente base empírica.

La ampliación de la escala geográfica tampoco aporta datos concluyentes. Las filiaciones entre el Cardial zonado y el Cardial tirrénico (Manen y Perrin 2009) siguen en estudio. La notable heterogeneidad de los distintos grupos ligures dificulta el establecimiento de grupos culturales amplios al oeste de Arene Candide (García-Atiénzar 2010). Los datos radiocarbónicos disponibles para el noroeste de Italia, Provenza y Languedoc hacen más difícil explicar la génesis del Cardial franco-ibérico y su relación cronológica con los distintos grupos precardiales (Manen y Sabatier 2003; Binder y Sénépart 2010). El problema reside en la escasez de muestras domésticas datadas de vida corta y en la complicada correlación de las fechas existentes con los yacimientos mejor estratificados.
En la zona ligur y provenzal con importantes secuencias revisadas y datadas puntualmente con muestras de vida corta, la coordinación entre las secuencias cultural, estratigráfica y radiocarbónica permite observar en parte la sustitución de lo impresso por lo cardial (Binder y Maggi 2001; Binder y Sénépart 2010). La situación relativa a ambos horizontes en el Languedoc, la zona de transición hacia la Península Ibérica, es más problemática. Faltan fechas de vida corta. Sitios, como el Abri de Jean Cros, con estratigrafías de calidad tienen dataciones, realizadas hace muchos años, con variaciones no asumibles. En otros, como la Grotte Gazel, se conocen los primeros datos sobre muestras de vida corta (Manen y Guilaine 2010) pero sus potentes secuencias estratigráficas están escasamente publicadas y/o en revisión. La evolución de la línea de costa ha destruido yacimientos litorales (Leucate-Corrège, les Dunes) (Guilaine et al. 1984; Carozza et al. 2005).

Esos problemas explican el lapso cronológico de unos 300 años entre los conjuntos impressa y los cardiales existentes en Languedoc. En cambio los estudios de los distintos materiales están muy avanzados, incluyendo análisis en profundidad (Manen et al. 2010). Por ese motivo, el incremento de dataciones radiocarbónicas a partir de taxones de vida corta procedentes de los contextos domésticos ya excavados facilitaría la comprensión del solapamiento de los grupos impressa y los cardiales. Tampoco se descarta que el vacío entre los dos horizontes se deba a una posible acción erosiva detectada en algunos yacimientos (Berger 2005) (Fig. 6).

En la zona provenzal, la coincidencia de fechas para las cerámicas cardiales no excluye la existencia de otras más antiguas como las de Grotte de Cap Ragnon (6480 $\pm 35 \mathrm{BP}$ sobre $\mathrm{Ca}$ pra), de Abri de Pendimoun Sep H2 (6450 \pm 40 BP sobre hueso humano) y de Balme d'Oullins c6 (6510 \pm 60 BP sobre Bos sp.) (Van Willigen et al. 2009; Binder y Sénépart 2010). A tenor de esos datos, cabría suponer que cualquier fecha anterior a c. $6500 \mathrm{BP}$ reflejaría un horizonte neolítico anterior y no un episodio cardial al occidente de la Provenza.

La información referente a las ocupaciones cardiales de la fachada oriental de la Península Ibérica, procedentes de niveles "aceptados" y con fechas radiocarbónicas antiguas, permiten poner en duda esa afirmación. Consideramos, en Cataluña, las de Les Guixeres de Vilobí (6655 \pm 45 BP, 


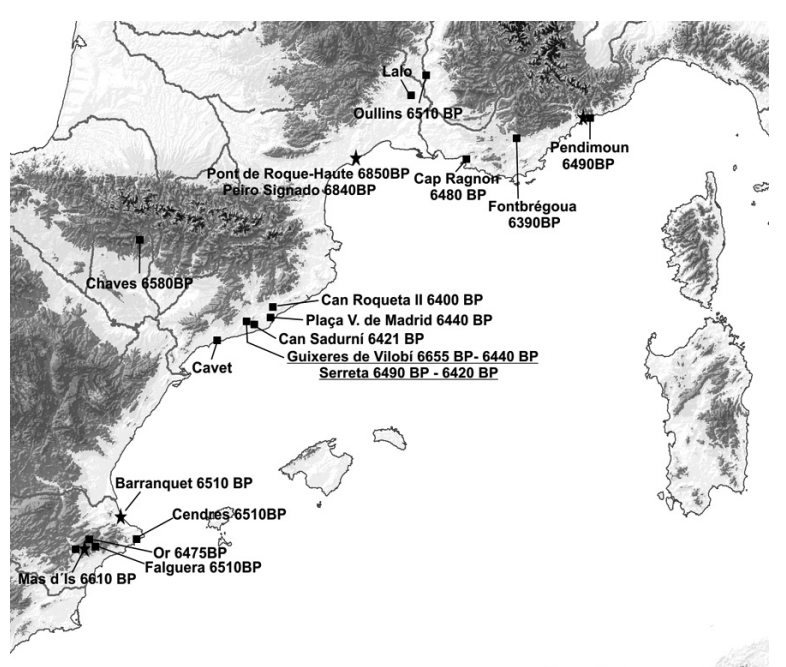

Fig. 6. Mapa de localización de los primeros grupos neolíticos en el noroeste mediterráneo. Las estrellas identifican los sitios con cerámica impressa y los cuadrados aquellos con cerámica cardial (subrayados los estudiados en el artículo).

en este trabajo) y las recientes fechas obtenidas sobre taxones domésticos en El Cavet; en el Alto Aragón las de la Cueva de Chaves Ib (6580 BP sobre Ovis aries) (Baldellou 2011); en Alicante la Cova de les Cendres H19 (6510 BP sobre Ovis aries), el Abric de la Falguera f.VI (6510 BP sobre Triticum monococcum) y la Cova de l'Or VIa (6475 BP sobre Ovis aries) (Bernabéu et al. 2009; Martí 2011).

Según los datos publicados, la explicación de la mayor antigüedad de las fechas cardiales de la Península Ibérica respecto a las del mediodía francés requiere conseguir otras nuevas en el territorio provenzal. Los datos radiocarbónicos referidos a los materiales de filiación impressa-ligur en la Península Ibérica son poco numerosos. Los materiales y fechas están bien caracterizados y analizados pero faltan precisiones estratigráficas. Nos referimos a las Casas 1 y 2 del Mas d'Is con fechas de $6600 \pm 50$ BP sobre Hordeum y materiales con estándares asimilables a los del sur de Italia (Bernabéu et al. 2009; Bernabéu et al. 2011); al Barranquet, con una de $6510 \pm 40 \mathrm{BP}$ para la UE79 sobre Ovis aries (Bernabéu et al. 2009), así como a la Cova d'en Pardo con otra de $6610 \pm 40$ BP para el nivel VIII sobre Ovis/ Capra (Soler et al. 2011). Los conjuntos cerámicos de los dos últimos son formalmente parecidos al horizonte ligur.
Falta en la Península Ibérica una secuencia continuada, al estilo de Pendimoun o Arene Candide, en asentamientos en cueva/abrigo o al aire libre que proporcione datos sobre las posibles relaciones o filiaciones cronológico-culturales. En los yacimientos donde aparecen materiales Impressa o hay un hiato hasta la primera ocupación Cardial (6400 BP en Mas d'Is y 6240 BP en Cova d'en Pardo) o falta la inmediatamente posterior (Barranquet). Además, las claras diferencias técnicas y temáticas entre las cerámicas de ambos grupos (Binder et al. 2010; McClure y Bernabéu 2011; Bernabéu et al. 2011) complican intuir la continuidad o filiación entre ambos tipos.

En este contexto, los datos en Cataluña (sin vestigios impressa de entidad) indican una rápida y amplia colonización por grupos cardiales tanto del valle del Llobregat (Penedès, Barcelona, Garraf y Vallès) como de la costa de Tarragona en cronologías cercanas a 6600 BP. Las numerosas excavaciones programadas y preventivas de los últimos años lo documentan mediante nuevos yacimientos y dataciones antiguas siempre integradas en el complejo Cardial (Martín et al. 2010).

Nuestra aportación, presentando registros arqueológicos fiables y dataciones radiocarbónicas para los yacimientos de Les Guixeres de Vilobí y La Serreta, ha permitido mejorar la integración de los datos acerca del Neolítico antiguo Cardial en la depresión del Penedès en el discurso actual sobre las primeras fases neolíticas. Los conjuntos cerámicos de dichos asentamientos son plenamente incorporables al grupo Cardial franco-ibérico con toda su gama clásica de matrices, formas y motivos decorativos. Además, su producción se ha podido situar entre 5600 y 5360 cal BC, ajustado a $1 \sigma$, en los silos de La Serreta y Les Guixeres A. Ello adscribe con claridad el conjunto del Penedès a la parte más antigua de la horquilla cardial y permite reabrir el debate, no exento de problemas y matices, sobre dónde y cuándo llegan los primeros grupos neolíticos al nordeste de la Península Ibérica. El avance en el estudio de los asentamientos neolíticos más antiguos y en su correcta contextualización cronológica proporcionará más datos al respecto.

\section{AGRADECIMIENTOS}

Este artículo se enmarca en el proyecto "Intervenció arqueològica en els enllaços a l'AP-7 a 
Vilafranca del Penedès", coordinado por el Institut Català d'Arqueologia Clàssica, financiado por la concesionaria ACESA y llevado a cargo por la empresa TRÍADE Serveis Culturals. Xavier Esteve y Xavier Oms llevan a cabo su investigación como miembros del grupo consolidado SGR200901145 del Seminari d'Estudis i Recerques Prehistòriques de la Universitat de Barcelona. Haidé Martins participa en el proyecto The Neolithic in the Mediterranean Basin, SFRH / BD / 44089 / 2008, financiado por Fundação para a Ciência e Tecnologia de la República Portuguesa, gracias al cual se obtuvieron las fechas radiocarbónicas de la Universidad de Oxford. La empresa ACESA ha sufragado las fechas radiocarbónicas de Beta Analytic. Ethel Allué (IPHES) es responsable de la identificación taxonómica de los carbones de La Serreta.

Jordi Farré del Vinseum - Museu de les Cultures del Vi de Vilafranca del Penedès-Catalunya nos prestó todas las facilidades para obtener las muestras de Les Guixeres de Vilobí que se dataron. Agradecemos a João Zilhão sus comentarios sobre las fechas radiocarbónicas y la discusión general.

Las correcciones realizadas por los revisores anónimos han enriquecido este trabajo.

\section{BIBLIOGRAFÍA}

Baldellou, V. 1979-1980: “Excavaciones arqueológicas en la Cova del Bolet (Mediona-Barcelona)". Pyrenae 15-16: 61-113.

Baldellou, V. 2011: “La Cueva de Chaves (BastarásCasbas, Huesca)". En J. Bernabéu, M. A. Rojo y Ll. Molina (eds.): Las primeras producciones cerámicas: el VI milenio cal AC en la Península Ibérica. Saguntum PLAV extra-12. Valencia: 141-144.

Baldellou, V. y Mestres, J. 1981: "Les Guixeres de Vilobí, un hàbitat del neolític antic a l'aire lliure". En El Neolitic a Catalunya Taula Rodona de Montserrat 1980: 69-74. Montserrat.

Baldellou, V.; Mestres, J.; Martí, M. y Juan-Cabanilles, J. 1989: El Neolítico antiguo. Los primeros agricultores en Aragón, Cataluña y Valencia. Diputación de Huesca. Huesca.

Bergadà, M. M.; Cebrià, A. y Mestres, J. 2005: “Prácticas de estabulación durante el Neolítico Antiguo en Cataluña a través de la micromorfología: Cueva de la Guineu (Font-Rubí, Alt Penedès, Barcelona)". En R. Ontañon, C. García-Moncó y P. Arias (eds.):
Actas del III Congreso del Neolítico en la Península Ibérica (Santander 2003): 187-196. Santander.

Berger, J. F. 2005: "Sédiments, dynamique du peuplement et climat au Néolithique ancien". En J. Guilaine (ed.): Populations néolithiques et environnements. Ed. Errance. Paris: 155-212.

Bernabéu, J. 1989: La tradición cultural de las cerámicas impresas en la zona oriental de la Península Ibérica. Serie de Trabajos Varios 86, Servicio Investigación Prehistórica. Valencia.

Bernabéu, J. 1999: "Pots, symbols and territories: the archaeological context of the neolithisations in Mediterranean Spain". Documenta Praehistorica XXVI: 101-118.

Bernabéu, J. y Molina, Ll. 2009: "La cerámica en la secuencia neolítica de Cendres". En J. Bernabéu y Ll. Molina (eds.): La Cova de les Cendres (Moraira-Teulada, Alicante). Serie Mayor 6, MARQMuseo Arqueológico de Alicante. Alicante: 55-84.

Bernabéu, J.; Molina, L1.; Esquembre, M. A.; Ortega, J. R. y Boronat, J. 2009: "La cerámica impresa mediterránea en el origen del Neolítico de la Península Ibérica". En De Méditerranéen et d'ailleurs... Mèlanges offerts à Jean Guilaine, Archives d'Ecologie Préhistorique. Toulouse: 83-95.

Bernabéu, J.; Gómez, O.; Molina, Ll. y García-Borja, P. 2011: "La cerámica neolítica durante el VI milenio AC en el Mediterráneo central peninsular". En J. Bernabéu, M. A. Rojo y Ll. Molina (eds.): Las primeras producciones cerámicas: el VI milenio cal AC en la Península Ibérica. Saguntum PLAV extra-12. Valencia: 153-178.

Binder, D.; Brochier, J. E.; Duday, H.; Helmer, D.; Marinval, P.; Thiébault, S. y Wattez, J. 1993: “L'Abri Pendimoun à Castellar (Alpes Maritimes): nouvelles données sur le complexe culturel de la céramique imprimée Méditerranéenne dans son contexte stratigraphique". Gallia Préhistoire 35: 177-251.

Binder, D.; Clop, X.; Convertini, F.; Manen, C. y Sénépart, I. 2010: "Les productions céramiques du Néolithique ancien entre Provence et Catalogne". En C. Manen, F. Convertini, D. Binder e I. Sénépart (eds.): Premières societés paysannes de Méditerrannée occidental. Structures des productions céramiques. Mémoire 51 Société Préhistorique Française. Toulouse: 115-132.

Binder, D. y Maggi, R. 2001: "Le Néolithique ancien de l'arc liguro-provençal". Bulletin de la Société Préhistorique Française 98, 3: 411-422.

Binder, D. y Sénépart, I. 2010: “La séquence de l'Impresso-Cardial de l'abri Pendimoun et l'evolution des assemblages céramiques en Provence". En C. Manen, F. Convertini, D. Binder e I. Sénépart (eds.): Premières societés paysannes de Méditerrannée occidental. Structures des productions céramiques. Mémoire 51 Société Préhistorique Française. Toulouse: 149-167. 
Bosch, J.; Chinchilla, J. y Tarrús, J. 2011: El poblat lacustre del neolitic antic de la Draga. Excavacions 2000-2005. Monografies del CASC 9, Museu d'Arqueologia de Catalunya-Centre d'Arqueologia Subaquàtica de Catalunya. Girona.

Carozza, J. M.; Odiot, T.; Carozza, L.; Jorda, C. y Pous, M. 2005: "Évolution paléogéographique de la plaine du Roussillon au cours de la seconde moitié de l'Holocène: implication sur la répartition des sites néolithiques". En J. F. Berger, F. Bertoncello, F. Braemer, G. Davtian y M. Gazenbeek (eds.): Teps et espaces de l'homme en société, analyses et modèles spatiaux en archéologie, XXVe rencontres internationales d'archéologie et d'histoire d'Antibes. Éditions APDCA. Antibes: 419-423.

Edo, M.; Blasco, A. y Villalba, M. J. 2011: "La Cova de Can Sadurní: guió sintètic de la prehistòria recent de Garraf'. En A. Blasco, M. Edo y M. J. Villalba (eds.): La cova de Can Sadurní i la prehistòria de Garraf. Actes de les Jornades Internacionals de Prehistòria 'El Garraf, 30 anys d'investigació arqueologica' (Begues 2008): 13-95. Milán.

Equip Guineu 1995: "Elaboració d'una cronoestratigrafia per a la Prehistòria del Penedès". Tribuna d'Arqueologia 1993-1994: 7-24.

Esteve, X.; Martin, P.; Oms, F. X.; Jornet, R. y López, D. 2012: "Intervencions arqueològiques als enllaços de l'autopista AP-7 de Vilafranca del Penedès: nous assentaments prehistòrics a l'aire lliure al Penedès". Tribuna d'Arqueologia 2010-2011: 23-39.

García-Atiénzar, G. 2010: "Las comarcas centromeridionales valencianas en el contexto de la Neolitización de la fachada noroccidental del Mediterráneo". Trabajos de Prehistoria 67 (1): 37-58.

Giró, P. 1947-1948: "Cuevas del Bolet (Mediona)". Ampurias IX-X: 261.

Guilaine J.; Freises A. y Montjardin R. 1984: LeucateCorrège, un habitat noyé du Néolithique cardial. Centre d'anthropologie des Sociétés rurales. Toulouse.

Manen, C. 2002: "Structure et identité des styles céramiques du Néolithique ancien entre Rhône et Èbre”. Gallia Préhistoire 44: 121-166.

Manen, C. y Guilaine, J. 2010: “Aspects géographiques et chronoculturels du Néolithique ancien languedocien". En C. Manen, F. Convertini, D. Binder e I. Sénépart (eds.): Premières societés paysannes de Méditerrannée occidental. Structures des productions céramiques. Mémoire 51 Société Préhistorique Française. Toulouse: 179-190.

Manen, C.; Convertini, F.; Binder, D. y Sénépart, I.: 2010: Premières societés paysannes de Méditerrannée occidental. Structures des productions céramiques. Mémoire 51 Société Préhistorique Française. Toulouse.

Manen, C. y Perrin, T. 2009: "Réflexions sur la genèse du Cardial 'franco-ibérique"'. En De Méditerra- née et d'ailleurs. Mélanges offerts à Jean Guilaine. Archives d'Écologie Prehistórique. Toulouse: 427-443.

Manen, C. y Sabatier, P. 2003: "Chronique radiocarbone la néolithisation en Méditerranée nord-occidental". Bulletin de la Societé Préhistorique Française 100, 3: 479-504.

Martí, B. 2011: "La Cova de 1'Or (Beniarrés, Alicante)". En J. Bernabéu, M. A. Rojo y Ll. Molina (eds.): Las primeras producciones cerámicas: el VI milenio cal $A C$ en la Península Ibérica. Saguntum PLAV extra-12. Valencia: 183-186.

Martín, A; Edo, M.; Tarrús, J. y Clop, X. 2010: “Le Néolithique ancien de Catalogne (VI - première moité du $\mathrm{V}$ millenaire av. J.C.). Les séquences chronoculturels". En C. Manen, F. Convertini, D. Binder e I. Sénépart (eds.): Premières societés paysannes de Méditerrannée occidental. Structures des productions céramiques. Mémoire 51 Société Préhistorique Française. Toulouse: 197-214.

McClure, S. y Bernabéu, J. 2011: “Technological style, chaîne opératoire, and labor investment of early Neolithic pottery". En J. Bernabéu, M. A. Rojo y Ll. Molina (eds.): Las primeras producciones cerámicas: el VI milenio cal AC en la Península Ibérica. Saguntum PLAV extra-12. Valencia: 53-60.

Mestres, J. 1981-82: "Avançament a l'estudi del jaciment de Les Guixeres de Vilobí". Pyrenae 17-18: 35-53.

Mestres, J. 1987: "La indústria lítica en sílex del neolític antic de Les Guixeres de Vilobí". Olerdulae 1-4: 5-71.

Mestres, J. 1992: "Neolitització i territorio". En 9è Col-loqui Internacional d'Arqueologia de Puigcerdà. Estat de l'investigació sobre el Neolític a Catalunya (Puigcerdà i Andorra la Vella 1991): 72-75. Puigcerdà.

Miró, J. M. 1992: "La fauna del jaciment de Les Guixeres de Vilobí (Alt Penedès) en el context del Neolític Antic a Catalunya". En 9è Col-loqui Internacional d'Arqueologia de Puigcerdà. Estat de l'investigació sobre el Neolític a Catalunya (Puigcerdà i Andorra la Vella 1991): 79-84. Puigcerdà.

Molina, Ll.; Bernabéu, J. y Orozco, T. 2011: “El Mas d'Is (Penáguila, Alicante)". En J. Bernabéu, M. A. Rojo y Ll. Molina (eds.): Las primeras producciones cerámicas: el VI milenio cal AC en la Península Ibérica. Saguntum PLAV extra-12. Valencia: 179-182.

Molist, M.; Bosch, J.; Clop, X.; Gómez, A. y Guerrero, E. 2009: "Des vases cardiales dans la ville de Barcelone: Apports du site de la Caserna de Sant Pau del Camp sur le néolithique ancien de la Catalogne". En De méditerranée et ailleurs... Mélanges offerts à Jean Guilaine. Archives d'Écologie Prehistórique. Toulouse: 547-555. 
Morales, J. I.; Fontanals, M.; Oms F. X. y Vergès, J. M. 2010: "La chronologie du Néolithique ancien cardial du nord-est de la Péninsule Ibérique. Datations, problématique et méthodologie". L'Anthropologie 114: 427-444.

Nadal, J.; Senabre, M. R., Mestres, J. y Cebrià, A. 1999: "Evolución del aprovechamiento de los recursos faunísticos durante el Neolítico en la comarca de l'Alt Penedès". En J. Bernabeu y T. Orozco (eds.): II Congrés del Neolític a la Península Ibèrica (València 1999): 85-94. Valencia.

Oms, F. X.; Petit, M. A.; Morales, J. I. y García, M. S. 2012: "Le processus de néolithisation dans les Pyrénées orientales. Occupation du milieu, culture matérielle et chronologie". Bulletin de la Société Préhistorique Française 109, 4: 651-670.
Soler, J. A.; Gómez, O.; García-Atiénzar, G. y Roca de Togores, C. 2011: "Sobre el primer horizonte neolítico de la Cova d'en Pardo (Planes, Alicante). Su evaluación desde el registro cerámico". En J. Bernabéu, M. A. Rojo, Ll. Molina (eds.): Las primeras producciones cerámicas: el VI milenio cal AC en la Península Ibérica. Saguntum PLAV extra-12. Valencia: 201-212.

Van Willigen, S.; Hajdas, I. y Bonani, G. 2009: "New radiocarbon dates for the early neolithic of the western Mediterranean". Radiocarbon 51, 2: 831-838.

Weninger, B.; Jöris, O. y Danzeglocke, U. 2011: CalPal-2007. Cologne Radiocarbon Calibration \& Palaeoclimate Research Package.

http://www.calpal.de/ 